\title{
Boundary Element Method for double diffusive natural convection in a horizontal porous layer
}

\author{
J. Kramer ${ }^{1}$, R. Jecl ${ }^{1} \&$ L. Škerget ${ }^{2}$ \\ ${ }^{1}$ Faculty of Civil Engineering, University of Maribor, Slovenia \\ ${ }^{2}$ Faculty of Mechanical Engineering, University of Maribor, Slovenia
}

\begin{abstract}
A numerical study of double-diffusive natural convection in porous media using the Boundary Element Method is presented. The studied configuration is a horizontal layer filled with fluid saturated porous media, where different temperature and concentration values are applied on the horizontal walls, while the vertical walls are adiabatic and impermeable. Transport phenomena in porous media are described with the use of modified Navier-Stokes equations in the form of conservation laws for mass, momentum, energy and species. The results for different governing parameters (Rayleigh number, Darcy number, buoyancy ratio and Lewis number) are presented and compared with those in published studies.

Keywords: Boundary Element Method, porous media, double-diffusive natural convection.
\end{abstract}

\section{Introduction}

Transport phenomena in porous medium is a subject of intensive research in last couple decades, mainly because of wide range of applications in many engineering branches. Problems of natural convection in porous media are most commonly studied examples. Many reported studies are dealing with natural convection driven by thermally buoyancy forces. A related problem that has received less attention is the so-called double-diffusive convection, where density differences occur due to combined thermal and compositional gradients across the porous layer. Some applications, where thermal natural convection or combined doublediffusive natural convection are observed, are fibrous insulation, geothermal energy, underground spreading of contaminants, solidification processes.

In horizontal layers, where horizontal walls are maintained at different temperatures and solute concentrations, the convective flow is possible above the 
critical Rayleigh number. In cases where the density differences are a result of combined temperature and concentration gradients, the critical Rayleigh number is a function of the Darcy number, Lewis number and buoyancy coefficient [1]. The flow structure in these cases becomes multi-cellular and is also called a Rayleigh-Benard flow structure [2]. Most of the studies regarding double-diffusive convection or thermohaline convection (the case where the constituent is salt) in a horizontal porous layers are focused on the problem of convective instability. There are many studies dealing with the onset of convection on the basis of linear stability theory [3, 4] or nonlinear perturbation theory [5]. In these studies the critical Rayleigh numbers for the onset of convective flows are predicted. The theoretical and numerical study of heat and mass transfer affected by a high Rayleigh number Benard convection in a porous layer heated from below is obtained in [6]. The numerical results and a scale analysis of the flow in a porous medium are presented, where the buoyancy effect is due entirely to temperature gradients.

Some further numerical results for a double-diffusive convection in a horizontal porous layer with two opposing buoyancy sources can be found in [7]. The influence of the governing parameters (Rayleigh number, Lewis number, buoyancy ratio) on the overall heat and mass transfer is discussed for the case of a square cavity. Double diffusive convection in a horizontal layer with some numerical results is also discussed in [8]. The critical values of Rayleigh numbers for the onset of convection are predicted on the basis of nonlinear parallel flow approximation.

All the above-mentioned numerical results are obtained on the basis of the Darcy flow model, which is more convenient for porous media with low permeability. The Brinkman extended Darcy model, on the other hand accounts for friction due to macroscopic shear is thus more appropriate when describing fluid flows in the porous matrix. This model was used in [1] to investigate the onset and development of double-diffusive convection in a horizontal porous layer with uniform heat and mass fluxes specified at the horizontal boundaries. The obtained analytical solutions are compared to some numerical results for different values of governing parameters.

The present study is focused on the development of the Boundary Element Method, for the problem of combined heat and mass transfer through horizontal porous layer. The Brinkman extended Darcy formulation is used to model the fluid flow in porous media, where the momentum equation is equivalent to the classical Navier-Stokes equations for pure fluid flow. The general set of equations is transformed with use of velocity-vorticity formulation, which consequently separates the numerical scheme into a kinematic and kinetic computational part [9].

\section{Mathematical formulation}

The configuration studied in the present paper is shown in fig. 1 .

It is a horizontal layer of width $D$ and height $H$, filled with homogenous nondeformable porous media, fully saturated with Newtonian fluid. The horizontal 


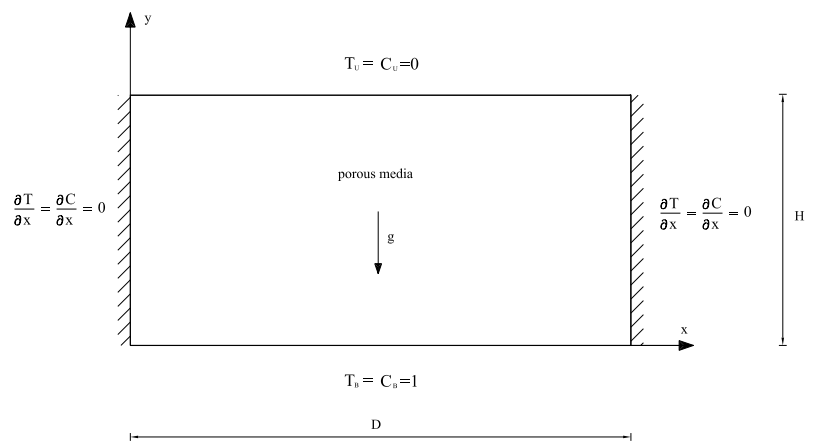

Figure 1: Geometry of a horizontal layer with boundary conditions.

walls are subjected to different temperature and concentration values $\left(T_{B}, C_{B}\right.$ at the bottom boundary and $T_{U}, C_{U}$ at the upper boundary), while the vertical walls are adiabatic and impermeable. The fluid saturating the porous media is modelled as a Boussinesq incompressible fluid, where the density depends only on temperature and concentration variations: $\rho=\rho_{0}\left(1-\beta_{T}\left(T-T_{0}\right)-\beta_{C}\left(C-C_{0}\right)\right)$, where the subscript 0 refers to a reference state, $\beta_{T}$ and $\beta_{C}$ are volumetric thermal and concentration expansion coefficients.

Transport phenomena in porous media is described using modified NavierStokes equations. The general set of macroscopic equations for conservation of mass, momentum, energy and species are written considering the fact that only a part of the volume, expressed with porosity $(\phi)$ is available for the flow of the fluid:

$$
\begin{gathered}
\frac{\partial v_{i}}{\partial x_{i}}=0 \\
\frac{1}{\phi} \frac{\partial v_{i}}{\partial t}+\frac{1}{\phi^{2}} \frac{\partial v_{j} v_{i}}{\partial x_{j}}=-\frac{1}{\rho_{0}} \frac{\partial p}{\partial x_{i}}+F g_{i}-\frac{\nu}{K} v_{i}+\frac{\partial}{\partial x_{j}}\left(2 \frac{\nu}{\phi} \dot{\varepsilon}_{i j}\right) \\
\frac{\partial}{\partial t}\left[\phi c_{f}+(1-\phi) c_{s}\right] T+c_{f} \frac{\partial v_{j} T}{\partial x_{j}}=\frac{\partial}{\partial x_{j}}\left(\lambda_{e} \frac{\partial T}{\partial x_{j}}\right) \\
\phi \frac{\partial C}{\partial t}+\frac{\partial v_{j} C}{\partial x_{j}}=\frac{\partial}{\partial x_{j}}\left(D \frac{\partial C}{\partial x_{j}}\right) .
\end{gathered}
$$

The parameters, used above are: $v_{i}$ volume-averaged velocity, $x_{i}$ the i-th coordinate, $\phi$ porosity, $t$ time, $\rho$ density, $\nu$ kinematic viscosity, $\partial p / \partial x_{i}$ the pressure gradient, $g_{i}$ gravity and $K$ permeability of porous media. $F$ is the normalized density difference function and is given as: $F=\left(\rho-\rho_{0}\right) / \rho_{0}=$ $-\left[\beta_{T}\left(T-T_{0}\right)+\beta_{C}\left(C-C_{0}\right)\right]$. Furthermore $c_{f}=(\rho c)_{f}$ and $c_{s}=(\rho c)_{s}$ are the heat capacities for the fluid and solid phases, respectively, $T$ is temperature, $\lambda_{e}$ the effective thermal conductivity of the porous media given as $\lambda_{e}=\phi \lambda_{f}+(1-\phi) \lambda_{s}$, where $\lambda_{f}$ and $\lambda_{s}$ are thermal conductivities for the fluid and solid phases, 
respectively. In the final equation $C$ stands for concentration, and $D$ for mass diffusivity. The momentum equation (2) is known as the Brinkman extension of the classical Darcy equation. The additional Brinkman viscous term (fourth on the r.h.s.) is analogous to the Laplacian term in the Navier-Stokes equations for pure fluid and accounts for viscous resistance or viscous drag force exerted by the solid phase on the flowing fluid at their contact surfaces.

\section{Boundary Element Method}

In the present study the extension of the classical Boundary Element Method (BEM) is used, the so called Boundary Domain Integral Method (BDIM) [9, 10]. Because in the obtained set of integral equations boundary and domain integrals are present, the discretization of surface and domain is required.

To use the BDIM the above given general set of equations should first be modified. Firstly, the modified velocity $v_{i}^{\prime}=v_{i} / \phi$ is introduced. The material properties, kinematic viscosity $\nu$ in the momentum equation, thermal diffusivity $a_{P}$ in the energy equation and mass diffusivity $D$ in the species equation are divided into a constant and variable part as follows: $\nu=\bar{\nu}+\tilde{\nu}, a_{P}=\bar{a}_{P}+\tilde{a}_{P}$ and $D=\bar{D}+\tilde{D}$. The momentum, energy and species equation can now be written as:

$$
\begin{gathered}
\frac{\partial v_{i}^{\prime}}{\partial t}+\frac{\partial v_{j}^{\prime} v_{i}^{\prime}}{\partial x_{j}}=-\frac{1}{\rho_{0}} \frac{\partial p}{\partial x_{i}}+F g_{i}-\frac{\nu \phi}{K} v_{i}^{\prime}+\bar{\nu} \frac{\partial^{2} v_{i}^{\prime}}{\partial x_{j} \partial x_{j}}+\frac{\partial}{\partial x_{j}}\left(2 \tilde{\nu} \dot{\varepsilon}_{i j}\right), \\
\frac{\sigma}{\phi} \frac{\partial T}{\partial t}+\frac{\partial v_{j}^{\prime} T}{\partial x_{j}}=\frac{\bar{a}_{P}}{\phi} \frac{\partial^{2} T}{\partial x_{j} \partial x_{j}}+\frac{\partial}{\partial x_{j}}\left(\frac{\tilde{a}_{P}}{\phi} \frac{\partial T}{\partial x_{j}}\right), \\
\frac{\partial C}{\partial t}+\frac{\partial v_{j}^{\prime} C}{\partial x_{j}}=\frac{\bar{D}}{\phi} \frac{\partial^{2} C}{\partial x_{j} \partial x_{j}}+\frac{\partial}{\partial x_{j}}\left(\frac{\tilde{D}}{\phi} \frac{\partial C}{\partial x_{j}}\right)
\end{gathered}
$$

where $\dot{\varepsilon}_{i j}$ represents the strain rate tensor $\dot{\varepsilon}_{i j}=1 / 2\left(\partial v_{i}^{\prime} / \partial x_{j}+\partial v_{j}^{\prime} / \partial x_{i}\right)$ and $\sigma$ is the heat capacity ratio given as $\sigma=\phi+(1+\phi) c_{s} / c_{f}$.

\subsection{Velocity-vorticity formulation}

In the next step the above-stated governing equations are transformed by the use of velocity-vorticity formulation (VVF), consequently the computational scheme is partitioned into its kinematic and kinetic parts [9].

In the present study a two-dimensional problem is considered, so all subsequent equations will be written for case of planar geometry.

The vorticity vector, which represents the curl of the velocity field $\omega=$ $e_{i j} \partial v_{j} / \partial x_{i}$ is introduced, where $e_{i j}$ is the unit permutation tensor. The kinematic part is represented by the elliptic velocity vector equation:

$$
\frac{\partial^{2} v_{i}^{\prime}}{\partial x_{j} \partial x_{j}}+e_{i j} \frac{\partial \omega^{\prime}}{\partial x_{j}}=0,
$$


where $\omega^{\prime}$ is the modified vorticity $\omega^{\prime}=\omega / \phi$. The kinetics is governed by the vorticity, energy and species transport equation. The vorticity transport equation is obtained as a curl of the Brinkman momentum equation (5):

$$
\frac{\partial \omega^{\prime}}{\partial t}+v_{j}^{\prime} \frac{\partial \omega^{\prime}}{\partial x_{j}}=\bar{\nu} \frac{\partial^{2} \omega^{\prime}}{\partial x_{j} \partial x_{j}}+e_{i j} g_{j} \frac{\partial F}{\partial x_{j}}-\frac{\nu \phi}{K} \omega^{\prime}+\frac{\partial}{\partial x_{j}}\left(\tilde{\nu} \frac{\partial \omega^{\prime}}{\partial x_{j}}\right)+\frac{\partial f_{j}}{\partial x_{j}}
$$

where $f_{j}$ is the contribution arising on account of nonlinear material properties. Equations (6), (7), (8) and (9) represent the leading non-linear set of equations, to which the weighted residual technique has to be applied. Integral representation of kinematic equation is:

$$
c(\xi) v_{i}^{\prime}(\xi)+\int_{\Gamma} v_{i}^{\prime} q^{*} d \Gamma=\int_{\Gamma} \frac{\partial v_{i}^{\prime}}{\partial n} u^{*} d \Gamma+\int_{\Omega} b_{i} u^{*} d \Omega,
$$

where the parameter $c(\xi)$ denotes coefficient related to the position of the source point. $b_{i}$ stands for the pseudo-body source term and is in this case $b_{i}=$ $e_{i j} \partial \omega^{\prime} / \partial x_{j}, u^{*}$ is the elliptic Laplace fundamental solution and $q^{*}$ is its normal derivative e.g. $q^{*}=\partial u^{*} / \partial n$. The fundamental solution $u^{*}$ for the case of planar geometry is given by the expression:

$$
u^{*}=\frac{1}{2 \pi} \ln \left(\frac{1}{r(\xi, s)}\right)
$$

where $r$ is the vector from the source point $\xi$ to the reference field point $s$. With further mathematical reformulations and use of Gauss-divergence theorem following integral formulation for kinematics can be written:

$$
c(\xi) v_{i}^{\prime}(\xi)+\int_{\Gamma} v_{i}^{\prime} q^{*} d \Gamma=e_{i j} \int_{\Gamma} v_{j}^{\prime} q_{t}^{*} d \Gamma-e_{i j} \int_{\Omega} \omega^{\prime} q_{j}^{*} d \Omega
$$

where $q_{t}^{*}$ is the tangential derivative of the fundamental solution $q_{t}^{*}=\partial u^{*} / \partial t$. The formulations for the vorticity, temperature and concentration can generally be written as a non-homogeneous elliptic diffusion-convective equation [10]:

$$
\bar{o} \frac{\partial^{2} u}{\partial x_{j} \partial x_{j}}-\frac{\partial \bar{v}_{j}^{\prime} u}{\partial x_{j}}-\frac{u}{\Delta t}+b_{i}=0
$$

where $u$ is taken as vorticity $\omega^{\prime}$, temperature $T$ and concentration $C$, respectively, $\bar{o}$ is defined by considering the conservation laws and constitutive hypothesis, and $b_{i}$ stands for the pseudo-body source term. Since the fundamental solution exists only for steady diffusion-convective PDE with constant coefficients, the velocity 
field is decomposed into an average constant vector $\bar{v}_{i}$ and perturbated vector $\tilde{v}_{i}$, such that $v_{i}=\bar{v}_{i}+\tilde{v}_{i}$. Thus the following integral formulation can be obtained:

$$
c(\xi) u(\xi)+\bar{o} \int_{\Gamma} u \frac{\partial u^{*}}{\partial n} d \Gamma=\int_{\Gamma}\left(\bar{o} \frac{\partial u}{\partial n}-u \bar{v}_{n}^{\prime}\right) d \Gamma+\int_{\Omega} b_{i} u^{*} d \Omega
$$

where $u^{*}$ is the elliptic diffusion-convective fundamental solution of steady diffusion-convective PDE, in the form of:

$$
u^{*}=\frac{1}{2 \pi \bar{o}} K_{0}(\mu r) \exp \left(\frac{\bar{v}_{j} r_{j}}{2 \bar{o}}\right) \text {, }
$$

for the plane case. Parameter $\mu$ is defined as $\mu=\sqrt{\left(\bar{v}^{\prime} / 2 \bar{o}\right)^{2}+\beta}$, where $\bar{v}^{\prime 2}=$ $\overline{v_{j}^{\prime} v_{j}^{\prime}}, \beta=1 / \bar{o} \Delta t, K_{0}$ is the modified Bessel function of the second kind of order 0 , and $r$ is the magnitude of the vector from the source to the reference point, i.e. $r=\left|x_{i}(\xi)-x_{i}(s)\right|$. The following integral representations for vorticity, temperature and concentration kinetics are obtained according to equation (14):

$$
\begin{aligned}
& c(\xi) \omega^{\prime}(\xi)+\int_{\Gamma} \omega^{\prime} \frac{\partial U_{\omega}^{*}}{\partial n} d \Gamma= \\
& =\frac{1}{\bar{\nu}} \int_{\Gamma}\left(\nu \frac{\partial \omega^{\prime}}{\partial n}-\omega^{\prime} v_{n}^{\prime}+e_{i j} g_{j} F n_{j}+f_{j} n_{j}\right) U_{\omega}^{*} d \Gamma+ \\
& +\frac{1}{\bar{\nu}} \int_{\Omega}\left(\omega^{\prime} \tilde{v}_{j}^{\prime}-e_{i j} g_{j} F-\tilde{\nu} \frac{\partial \omega^{\prime}}{\partial x_{j}}-f_{j}\right) \frac{\partial U_{\omega}^{*}}{\partial x_{j}} d \Omega+ \\
& +\frac{1}{\bar{\nu}} \int_{\Omega} \frac{\nu \phi}{K} \omega^{\prime} U_{\omega}^{*} d \Omega+\frac{1}{\bar{\nu} \Delta t} \int_{\Omega} \omega_{F-1}^{\prime} U_{\omega}^{*} d \Omega, \\
& c(\xi) T(\xi)+\int_{\Gamma} T \frac{\partial U_{T}^{*}}{\partial n} d \Gamma=\frac{\phi}{\sigma \bar{a}_{P}} \int_{\Gamma}\left(\frac{\sigma a_{P}}{\phi} \frac{\partial T}{\partial n}-T v_{n}^{\prime}\right) U_{T}^{*} d \Gamma- \\
& -\frac{\phi}{\sigma \bar{a}_{P}} \int_{\Gamma}\left(\frac{\sigma \tilde{a}_{P}}{\phi} \frac{\partial T}{\partial x_{j}}-T \tilde{v}_{j}^{\prime}\right) \frac{\partial U_{T}^{*}}{\partial x_{j}} d \Gamma+ \\
& +\frac{\phi}{\sigma \bar{a}_{P} \Delta t} \int_{\Omega} T_{F-1} U_{T}^{*} d \Omega, \\
& c(\xi) C(\xi)+\int_{\Gamma} C \frac{\partial U_{C}^{*}}{\partial n} d \Gamma=\frac{\phi}{\bar{D}} \int_{\Gamma}\left(\frac{D}{\phi} \frac{\partial C}{\partial n}-C v_{n}^{\prime}\right) U_{C}^{*} d \Gamma- \\
& -\frac{\phi}{\bar{D}} \int_{\Omega}\left(\frac{\tilde{D}}{\phi} \frac{\partial C}{\partial x_{j}}-C \tilde{v}_{j}^{\prime}\right) \frac{\partial U_{C}^{*}}{\partial x_{j}} d \Omega+ \\
& +\frac{\phi}{\bar{D} \Delta t} \int_{\Omega} C_{F-1} U_{C}^{*} d \Omega,
\end{aligned}
$$

where $U_{\omega}^{*}, U_{T}^{*}$ and $U_{C}^{*}$ are modified elliptic diffusion-convective solutions defined as $U_{\omega}^{*}=\bar{\nu} u^{*}$ in the momentum equation, $U_{T}^{*}=\bar{a}_{P} / \phi u^{*}$ in the energy equation and $U_{C}^{*}=\bar{D} / \phi u^{*}$ in the species equation. 
For approximate numerical solution, the integral equations have to be written in a dicretized manner, where the integrals over boundary and domain are approximated by the sum of integrals over all boundary elements and internal cells, respectively. The variation of field functions within each boundary element or internal cell is approximated by the use of appropriate interpolation polynomials [11]. The system of discretized equations is solved by coupling those kinetic and kinematic equations and considering the corresponding boundary and initial conditions. Since the implicit set of equations is written simultaneously for all boundary and internal nodes, this procedure results in a very large and fully-populated system matrix, influenced by diffusion and convection. The consequence of this approach is a very stable and accurate numerical scheme with substantial computer time and memory demands. The subdomain technique is used to improve the economics of the computation, where the entire computational domain is partitioned into subdomains to which the same described numerical procedure can be applied [9].

\section{Test example}

The obtained numerical scheme is discussed on the problem described in section 2 of this paper. The governing parameters of a problem are:

- porosity, $\phi$,

- Darcy number $D a$, given with the expression $D a=K / \phi H^{2}$

- aspect ratio $A=D / H$

- modified (porous) thermal Rayleigh number $R a=K g \beta_{T} \Delta T H / a_{P} \nu$,

- Lewis number $L e=a_{P} / D$,

- buoyancy ratio $N=\beta_{C} \Delta C / \beta_{T} \Delta T$.

In the above notations $\Delta T$ and $\Delta C$ are temperature and concentration differences between the upper and bottom boundaries, and $D$ is the mass diffusivity. For aspect ratio $A=1$, a non-uniform computational mesh $20 \times 20$ subdomains was used with a ratio between the longest and shortest elements of $r=6$, and for $A=2, A=4$ $20 \times 10$ subdomains were used. Time-steps ranging from $\Delta t=10^{-16}$ (steady state) to $\Delta t=10^{-4}$ were employed, and the convergence criterion is determined as $\varepsilon=5 \times 10^{-6}$ for all cases.

The described numerical model was tested for different values of governing parameters $(R a, D a, L e$ and $N$ ). It should be noted that, in the case of $N=0$ the buoyancy effect is due entirely to temperature gradients. The mass transfer in this case is due to temperature field and concentration differences between the horizontal boundaries. In the case of positive values for buoyancy ratio $(N>0)$, the thermal and solutal buoyancy forces aid each other (aiding convection) and for negative values of buoyancy ratio $(N<0)$ the solutal and thermal effects have opposite tendencies (opposing convection).

The results for total heat and mass transfer through the horizontal layer are given by the values of Nusselt and Sherwood numbers defined as:

$$
N u=-\int_{0}^{1}\left(\frac{\partial T}{\partial x}\right)_{x=0} d y, \quad S h=-\int_{0}^{1}\left(\frac{\partial C}{\partial x}\right)_{x=0} d y .
$$


Table 1: Comparison of results with numerical experiments reported in the literature for $A=1$.

\begin{tabular}{ccccccc}
\hline & \multicolumn{3}{c}{$N u$} & & \multicolumn{2}{c}{$S h$} \\
\cline { 2 - 3 } \cline { 5 - 6 } \cline { 5 - 6 } & This Study & Ref. [7] & & This study & Ref. [7] \\
\hline$R a=600, L e=1, N=0$ & 7.01 & 6.6 & & 7.01 & - \\
$R a=100, L e=10, N=0.2$ & 2.48 & 2.4 & & 10.00 & - \\
$R a=100, L e=10, N=0.2$ & 2.50 & 2.5 & & 14.8 & 15 \\
\hline
\end{tabular}

The validation of the numerical code was accomplished by comparison with some published numerical experiments. In table 1 some results for $A=1, D a=10^{-5}$, different values of Rayleigh, Lewis numbers and buoyancy ratio are presented.

The values of overall heat and mass transfer are compared with the published results, where the numerical calculations based on the Darcy model are obtained [7]. The first result is for the case of $R a=600, L e=1$ and $N=0$, which means, that only the thermal buoyancy force is present. The overall heat and mass transfer, which are presented by $N u$ and $S h$ are identical. The other two cases are for $R a=100, N=0.2$ and $L e=10, L e=30$. In this case, both the thermal and solutal buoyancy forces are present and aid each other. The values of Sherwood numbers are now higher than those of the Nusselt numbers, which is a result of higher Lewis number. The presented result are in agreement with published ones. Table 2 presents the influence of the Darcy number on the overall heat and mass transfer. The values of the governing parameters for this case are: aspect ratio $A=4$, Rayleigh number $R a=300$, Lewis number $L e=0.1$ and buoyancy ration $N=-2$. The negative sign for buoyancy ratio indicates, that the thermal and solutal buoyancy forces oppose to each other.

Table 2: $N u$ and $S h$ numbers for different values of $D a$ and $A=4, R a=300$, $L e=0.1, N=-2$.

\begin{tabular}{cccccc}
\hline$D a$ & $10^{-1}$ & $10^{-2}$ & $10^{-3}$ & $10^{-4}$ & $10^{-5}$ \\
\hline$N u$ & 1.00 & 2.05 & 2.82 & 3.12 & 3.50 \\
$S h$ & 1.00 & 1.02 & 1.04 & 1.05 & 1.06 \\
\hline
\end{tabular}

From the obtained results it is evident, that with any decrease in the Darcy number the vaule of the Nusselt number increases. In cases of small Lewis numbers $L e \rightarrow 0$ the values of Sherwood numbers tend to unity $(S h \rightarrow 1)$, which implies, that the mass transfer is dominated by diffusion. The same conclusions are also 
published in [1]. In the case of $D a=10^{-1}$ the values of $N u$ and $S h$ are equal to 1 , which means that heat and mass transfer are governed by diffusion. The Rayleigh number in this case is beyond the critical value required for the beginning of convective motion. The relationship between the critical Rayleigh number and the Darcy number is given in [1] and states that the values of the critical Rayleigh number increase in line with the Darcy number.

The velocity, temperature and concentration fields for $R a=2500, D a=10^{-2}$, $L e=10, N=0, A=2$ are presented in fig. 2 .

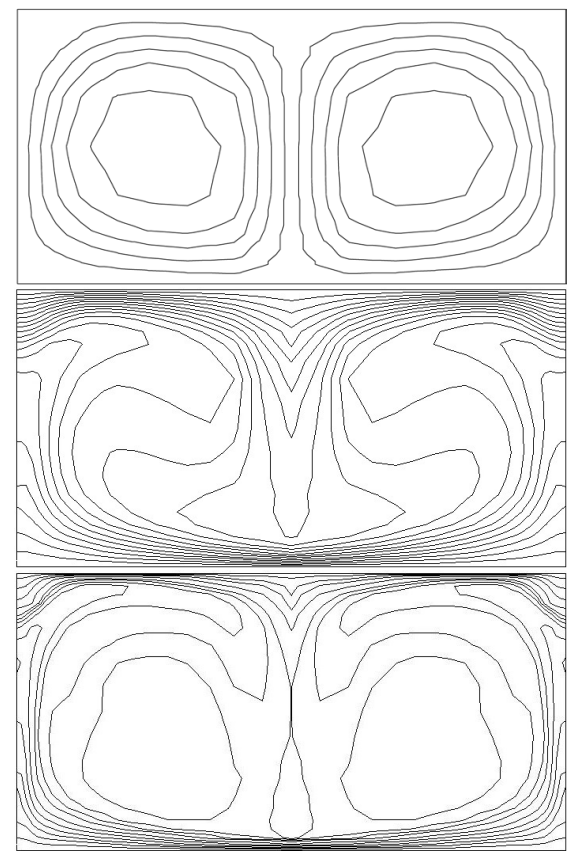

Figure 2: Streamlines, isotherms and isoconcentrations in a horizontal layer for $R a=2500, D a=10^{-2}, L e=10, N=0, A=2$.

The buoyancy effect in this case is due entirely to temperature gradients, so the concentration field is a result of the flow driven by the temperature gradients and the imposed concentration difference between the upper and bottom boundaries. From the fig. 2. it is evident that the flow in the horizontal layer with $A>1$ becomes multi-cellular (in the case of $A=2$ there are 2 cells). The flow consists of rising hot fluid in the centre of the layer and colder fluid sinking along the vertical walls. In the centre of the domain higher solute concentration is found than along the adiabatic and impermeable side walls. Thin temperature and composition boundary layers are evident at the top and bottom walls. 


\section{Conclusion}

A numerical study of double-diffusive natural convection in a horizontal porous layer, saturated with the Newtonian and incompressible fluid is presented. For the solution of governing equations the Boundary Domain Integral Method, an extension of the classical Boundary Element Method, was used. The modified Navier-Stokes equations have been used to describe the fluid motion in porous media. The general set of equations is transformed with use of velocity-vorticity formulation, which consequently separates the computational scheme into a kinematic and kinetic part. The results for different values of governing parameters are obtained and compared to some published studies.

\section{References}

[1] Amahmid, A., Hasnaoui, M., Mamou, M. \& Vasseur, P., Double-diffusive parallel flow induced in a horizontal brinkman porous layer subjected to constant heat and mass fluxes: analytical and numerical studies. Heat and Mass Transfer, 35, pp. 409-421, 1999.

[2] Kladias, N. \& Prasad, V., Natural convection in horizontal porous layers: Effects of darcy and prandtl numbers. Journal of Heat Transfer, 111, pp. 926935, 1989.

[3] Nield, D.A., Onset of thermohaine convection in a porous medium. Water Resour Res, 4, pp. 553-560, 1968.

[4] Nield, D.A., Manole, D.M. \& Lage, J.L., Convection induced by inclined thermal and solutal gradients in a shallow horizontal layer of a porous medium. J Fluid Mech, 257, pp. 559-574, 1993.

[5] Rudraiah, N., Srimani, P.K. \& Friedrich, R., Finite amplitude convection in a two-component fluid saturated porous layer. Int J Heat Mass Transfer, 25, pp. 715-722, 1982.

[6] Trevisan, O.V. \& Bejan, A., Combined heat and mass transfer by natural convection in a vertical enclosure. Journal of Heat Transfer, 109, pp. 104$112,1987$.

[7] Rosenberg, N.D. \& Spera, F.J., Thermohaline convection in a porous medium heated from below. Int J Heat Mass Transfer, 35, pp. 1261-1273, 1992.

[8] Mamou, M., Vasseur, P. \& Bilgen, E., Multiple solutions for double diffusive convection in a vertical porous enclosure. Int J Heat Mass Transfer, 38, pp. 1787-1798, 1995.

[9] Škerget, L., Hriberšek, M. \& Kuhn, G., Computational fluid dynamics by boundary-domain integral method. Int J Numer Meth Engng, 46, pp. 1291$1311,1999$.

[10] Jecl, R. \& Škerget, L., Boundary element method for natural convection in non-newtonian fluid saturated square porous cavity. Engineering Analysis with Boundary Elements, 27, pp. 963-975, 2003.

[11] Brebbia, C.A. \& Dominguez, J., Boundary Elements, An Introductory Course. McGraw-Hill Book Company, New York, 1992. 